\title{
Lumen
}

Selected Proceedings from the Canadian Society for Eighteenth-Century Studies

\section{Un nouveau jour brille sur l'horizon : le temps dans la pédagogie de la Révolution}

\section{Nancy Senior}

Volume 18, 1999

Representations of Time in the XVIIIth Century

Le temps et ses représentations au dix-huitième siècle

URI : https://id.erudit.org/iderudit/1012371ar

DOI : https://doi.org/10.7202/1012371ar

Aller au sommaire du numéro

Éditeur(s)

Canadian Society for Eighteenth-Century Studies / Société canadienne d'étude du dix-huitième siècle

ISSN

1209-3696 (imprimé)

1927-8284 (numérique)

Découvrir la revue

Citer cet article

Senior, N. (1999). Un nouveau jour brille sur l'horizon : le temps dans la pédagogie de la Révolution. Lumen, 18, 119-134.

https://doi.org/10.7202/1012371ar

Copyright (C Canadian Society for Eighteenth-Century Studies / Sociéte canadienne d'étude du dix-huitième siècle, 1999
Ce document est protégé par la loi sur le droit d'auteur. L'utilisation des services d'Érudit (y compris la reproduction) est assujettie à sa politique d'utilisation que vous pouvez consulter en ligne.

https://apropos.erudit.org/fr/usagers/politique-dutilisation/ 


\section{Un nouveau jour brille sur l'horizon: le temps dans la pédagogie de la Révolution}

Les partisans de la Révolution française voient souvent les événements en termes d'un nouveau jour. C'est une métaphore à double face: d'un côté, la nouveauté, un commencement, toutes les possibilités ouvertes; de l'autre, un cycle, une récurrence, la répétition de ce qui est déjà arrivé tant de fois. Ce nouveau jour est représenté dans le texte et dans l'iconographie d'œuvres pédagogiques de la Révolution, ainsi que dans le calendrier républicain, qui lui aussi a été conçu comme un instrument d'instruction. ${ }^{1}$ Les œuvres pédagogiques dont je parlerai ici, et dont quelques-unes sont imprimées, d'autres manuscrites, ont été composées pour le concours de livres scolaires ouvert sous la Convention par une loi du 8 pluviôse, an II - à un temps de changements rapides de la situation politique. Le Comité d'Instruction publique a demandé des livres élémentaires dans plusieurs domaines, y compris le soin physique des petits enfants, la lecture, la grammaire française, les mathématiques, la géographie et la morale républicaine. Des auteurs distingués y ont pris part, comme Condorcet pour les mathématiques, De Wailly pour la lecture et l'orthographe, Lhomond pour la grammaire, Volney pour la morale. ${ }^{2} C^{\prime}$ est dans cette dernière catégorie — la morale — qu'il y a eu le plus de soumissions, de qualité très inégale et à l'orthographe souvent incertaine (reproduite dans les citations plus bas). ${ }^{3}$ Ces textes, provenant de différentes parties de la France et composés par des personnes d'origines sociales diverses, constituent un témoignage des idées de Français dont beaucoup sont éloignés de l'élite parisienne. ${ }^{4}$

Le calendrier est au contraire le produit d'une commission centrale; il a été conçu dans le but de faire pour le temps ce que les nouveaux poids et mesures ont fait pour les corps physiques. Il doit marquer un temps nouveau, libéré des entraves de l'histoire et des erreurs scientifiques et religieuses. Nous verrons dans quelle mesure ce calendrier correspond à certaines idées qu'on trouve dans les écrits pédagogiques. 


\section{Un nouveau jour}

La métaphore de la lumière, souvent utilisée par le christianisme, avait été appropriée par le mouvement philosophique - d'où les expressions bien connues comme 'le siècle des Lumières', 'die Aufklärung', 'the Enlightenment.' Les écrivains 'philosophes' du XVIIIe siècle font appel à tout un réseau de significations: raison - lumière - nature - universel. La raison se sert de ses lumières pour trouver ce qui est naturel, et qui est supposé aussi être universel. Toutes ces idées se trouvent rassemblées dans le frontispice du Catéchisme de la nature de Pierre-Platon Blanchard. ${ }^{5}$

Au milieu de ce tableau, on voit la Nature ou l'Abondance, aux quatre seins. Un génie ailé (on n'ose plus dire un ange, à cette époque) indique d'une main le livre ouvert, et de l'autre main la déesse. Au fond à droite, le soleil se lève, dissipant la nuit et les nuages de l'obscurantisme. On voit de petits personnages, partisans des ténèbres, qui s'éloignent furtivement. Au premier plan, des représentants des continents: l'Asie, l'Amérique, le Moyen Orient, l'Afrique et l'Europe, admirent la merveille qu'on leur montre. La légende en bas invite le spectateur: '[v]oulez-vous être heureux? écoutez la Nature.' Nous voyons que la nature et la raison sont révélées à toute la terre, à toute l'humanité.

Dans les ouvrages pédagogiques de la Révolution et spécifiquement ceux présentés au concours, le changement de régime est souvent représenté comme le triomphe de la nature et de la raison. Renaud dit à son élève: '[c]onsidere le gouvernement Republicain comme le plus juste, le plus naturel, \& le plus conforme à notre destinée terrestre. ${ }^{6}$ Les principes de la République sont l'œuvre de Dieu: 'Dieu a créé les hommes libres et égaux. ${ }^{7}$ Dieu a donné à l'homme 'la raison, ce flambeau pur de sa lumière, cette étincelle de sa divinité, pour diriger ses actions. ${ }^{\prime 8}$ La conscience a besoin d'être éclairée par 'le flambeau de la raison', dit Levesque dans son Cours élémentaire de morale. ${ }^{9}$ Pour bien utiliser la raison, il faut être instruit, car l'ignorance est la cause de toutes les maladies de l'âme, comme le formule l'auteur anonyme d'un manuscrit intitulé Instruction élémentaire sur la morale républicaine: '[la] raison doit éclairer votre volonté et diriger vos actions. ${ }^{10}$ Les prêtres ont empêché la lumière de la raison de briller pour tout le monde: 'si quelques étincelles de raison échappaient, ils nous ordonnaient d'en faire le sacrifice. ${ }^{11}$ Cela ne veut pas dire nécessairement qu'on doit éviter de parler de Dieu; au contraire, un père doit en parler à son fils 'lorsqu'il remarque en lui la premiere étincelle de la raison' (Blanchard 16).

Avec la Révolution, selon nos auteurs, la raison entre finalement dans ses droits. La citoyenne Cavaillon dit qu'elle est 'heureuse si en combattant d'anciens préjugés, enfans de l'orgeüil, du mensonge et de l'igno- 


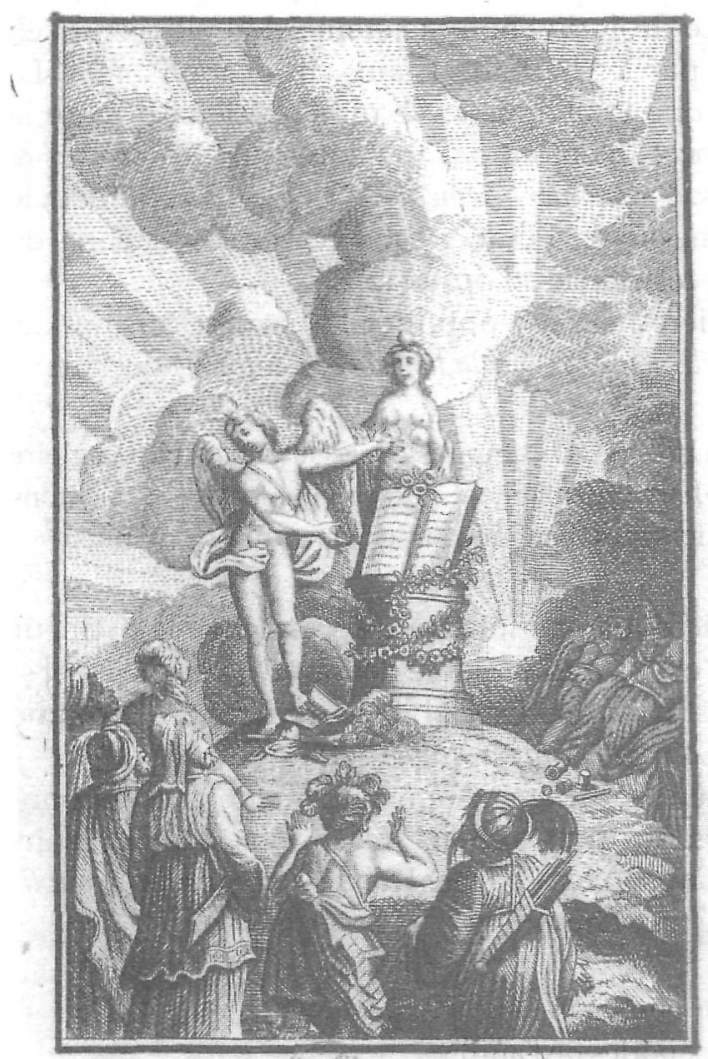

Frontispice du Catéchisme de la nature de Pierre-Platon Blanchard (cliché Bibliothèque nationale de France).

Toulew vous cilen heuraux? ćcontex la Nature

rance, [elle peut] porter un rayon de lumiere..$^{12}$ Desparens veut animer les 'ames neuves et pures' des élèves du 'feu sacré de la liberté. ${ }^{13}$ Collignon, dans L'Instituteur du peuple, ou La vérité sans voile, écrit en épigraphe les vers suivants:

Lisez \& frémissez vils esclaves des rois, L'auguste vérité vous range sous ses loix Le despote en frémit \& et la nature entiere Eclaire le français de sa douce lumiere. ${ }^{14}$

Par contraste avec ce présent lumineux, le passé est peint comme un temps de ténèbres. Dans un des dialogues de la citoyenne Cavaillon, la bonne mère dit à son fils: 'tu apprendras en fremissant, que pendant 
plusieurs siécles, tes ayeux ont porté des fers, et gemi sous le gouc honteux de lesclavage.' Le droit de l'homme était, selon elle, 'enseveli dens la nuit des sciécles par les barbares mains du despotisme. ${ }^{15} \mathrm{La}$ citoyenne Desmarest dit que la liberté est 'le plus précieux don de la nature', mais que les Français n'en ont pas toujours joui; 'les Prêtres \& les Rois nous l'avoient ravie' (Premiers éléments 12). Selon Taboureau, le premier devoir de l'homme envers l'État, 'c'est d'ecarter les ténèbres de l'imposture en oposant la résistance a l'opression. ${ }^{\prime 16}$

La France est la première nation à apporter la lumière au monde. L'auteur des Instructions élémentaires sur la morale républicaine déclare:

il semble que la divinité, qui préside aux empires, réservait à la france la gloire d'entreprendre, la premiere le renouvellement du monde moral. [...] félicitons nous donc de jouir, les premiers, des bienfaits d'une Constitution sublime. ${ }^{17}$

Il était peut-être inévitable que le peuple français établisse le meilleur des gouvernements, car c'est 'le plus sociable de tous les peuples [...] si l'homme comme on dit est un animal sociable, le français est l'homme par excellence' (Prévost, Education indispensable 120).

Les images de la lumière se trouvent non seulement dans l'iconographie et dans les textes des traités, mais aussi dans des chansons révolutionnaires qui en font souvent partie. Dans l'Evangile des républicains, $\mathrm{l}^{\prime \prime}$ Hymne à la liberté' proclame:

Un nouveau jour brille sur l'horizon.

Des rives du couchant, aux portes de l'aurore,

Pour la première fois, l'Univers se colore

Du feu sacré de la raison. ${ }^{18}$

Dans 'Un père à son fils', le père appelle son fils en lui disant: '[r]éveilletoi, voici l'aurore, / Mon fils, voici ton plus beau jour' (95). Un autre hymne est adressé au soleil, qu'on appelle

Astre, père du monde, ô roi dont la lumière,

En embrassant l'immensité

Eclaira jusqu'ici dans ta vaste carrière,

La terre de la liberté [...].

Dans cette même chanson, on dit que

Le Français a brisé ses fers:

Il a puni, dans sa justice,

Les oppresseurs de l'Univers, 
Et les tyrans du saint office,

Au premier cri de la raison.

Du sérail de Byzance, au conclave de Rome,

De l'Africain, jusqu'au Lapon,

Le globe a retenti des Droits sacrés de l'homme. (72-73)

Cette chanson exprime bien l'état d'esprit de la gravure que nous avons décrite, où le livre de la nature et de la raison est ouvert à toute l'humanité.

\section{Présent, passé, futur}

Pourtant la Révolution, comme on le sait bien, n'est pas représentée par ses fervents défenseurs seulement comme un avenir lumineux. On la présente aussi comme le retour à un passé idéalisé, à une antiquité mythique, produit de plusieurs siècles d'éducation dans les collèges où les jeunes Français lisaient des textes latins choisis pour leur inspirer une vertu héroïque. Une autre gravure qui sert de frontispice à deux œuvres de Fréville, toutes deux soumises au concours (Le Temple de la morale et Vie et mort républicaines $d u$ petit Emilien) illustre bien cette tendance de retour au passé. ${ }^{19}$ La gravure représente un monument à Emilien, le fils de l'auteur, mort à l'âge de six ans. On y remarque l'iconographie de l'antiquité, si fréquente à l'époque: cyprès, bonnet phrygien, faisceaux de verges, que portait le licteur romain. Ce désir de retrouver l'antiquité pourrait faire penser à une conception cyclique du temps. En bas de la gravure, la légende nous informe que le petit Emilien est 'Né le 24 8re 1786 et mort le 22. Br. An 2. R.F.' Cet enfant est donc né pendant une ère, qu'on appelle l'ère vulgaire, et mort pendant une autre, l'ère républicaine. La nouvelle façon de compter les ans suggère un tout nouveau commencement, un temps linéaire. A la différence de la première gravure que j'ai décrite, celle-ci ne contient rien qui représente l'humanité entière; au contraire, il n'y a que la patrie qui compte. Deux petits anges tristes assis sur le monument portent des rouleaux sur lesquels on lit: '[1]a patrie est tout. Tout pour la patrie.' La tonalité de cette gravure, ainsi que son sens, est en fort contraste avec l'autre; les symboles funéraires abondent, et l'enfant, qui représente l'avenir, est mort. Pourtant, il est mort en enfant de la République, un modèle qu'on propose pour inspirer les mêmes sentiments chez d'autres enfants qui, eux, vivront pour la patrie.

Cette gravure nous mène à une autre question. Dans une révolution où on essaie de changer non seulement le régime, mais aussi les mentalités de toute une nation, que devient le rapport entre parents ou 


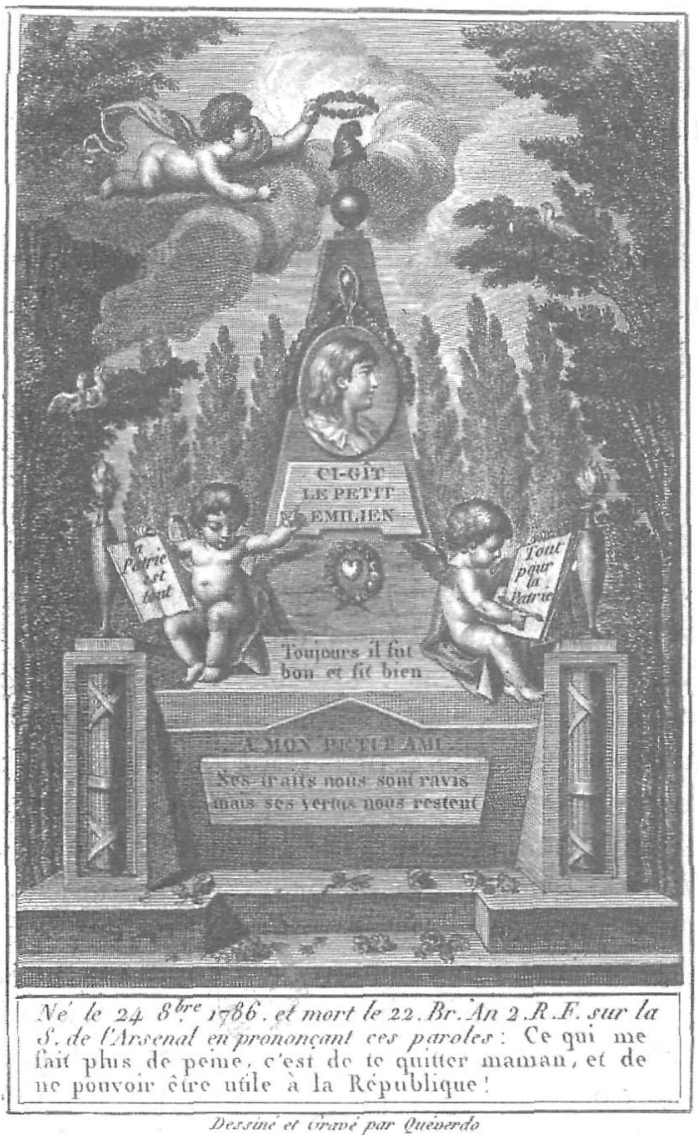

Gravure du petit Emilien (cliché Bibliothèque nationale de France).

maîtres d'école et enfants, ou autrement dit, entre la tradition et l'avenir? Nous avons vu dans 1'Hymne au soleil' que le soleil est interpellé comme un père et un roi. Les défenseurs de la monarchie avaient en effet l'habitude de la justifier en comparant le roi à un père de famille. Or, à une époque où il n'y a plus de roi en France, la chute de la monarchie entraîne-t-elle l'affaiblissement de l'autorité parentale?

Les opinions des auteurs de livres scolaires présentés au concours sont très diverses sur la question de l'autorité. Certains suivent la tradition qui veut que l'enfant soit obéissant et docile. Selon Godineau:

[1]'enfant qu'on n'ose, ni punir, ni corriger, est bien à plaindre. C'est un enfant gâté qui reprochera un jour amèrement à ses père et mère, $l^{\prime}$ indulgence dont ils auront usé à son égard [...]. Veux-tu connoître tes devoirs? lis-les sur le visage de tes parens [...]. Le premier des devoirs d'un enfant, est l'obéissance. ${ }^{20}$ 
La citoyenne Lambert, institutrice, déclare: '[o]n voit briller la sagesse dans un Enfant docile et doux, celui qui est mutin et désobéissant, se fait haïr de ses maîtres et de ses Camarades' (Avis aux jeunes républicaines françoises, $21^{\mathrm{e}}$ maxime). La bonne mère dans les dialogues de la citoyenne Cavaillon dit à son fils: 'si tu veus etre digne de la république, il faut t'accoutumér de bonne heurs, a etre obeissant a tes superieurs.' Prévost considère que même les parents qui ont des défauts méritent le respect et l'obéissance des enfants:

Honore ton père de tout ton cœur, et n'oublie pas les douleurs que ta mère a souffertes [...] soulage-les dans la vieillesse [...] obéis-leur. - Mais ce père est méchant: qu'importe! la nature t'a-t-elle nécessairement lié à un bon père? non, mais à un père. ${ }^{21}$

Quelques auteurs au contraire disent, en citant souvent Rousseau, que les jeunes enfants doivent être libres. La citoyenne Guérin-Albert applique ce principe de façon plus conséquente que le maître, car elle désire que les filles aussi bien que les garçons jouissent de la liberté. Jusqu'à six ans, dit-elle, 'ma fille fera ou ne fera pas la révérence, dira ou ne dira pas bonjour ou bonsoir, je ne gênerai nullement sa volonté. ${ }^{22} L^{\prime}$ usage des punitions est condamné par de nombreux auteurs. Aubert se plaint des 'châtiments honteux' dont on use dans les collèges. Ces châtiments flétrissent l'innocence des enfants, et les habituent à 's'asservir à l'opinion d'autrui. ${ }^{, 23}$ Cyrille Rigaud, docteur en médecine, lui aussi s'élève contre l'idée selon laquelle '[q]ui aime bien, bien châtie'. Selon Rigaud, '[c]e que l'enfant ne saurait apprendre sans châtiment, il ne doit pas le savoir, il n'est pas organisé pour cela. ${ }^{24}$ Mais ces auteurs, comme les autres, supposent que l'enfant finira par apprendre ce que les adultes veulent qu'il sache. Même l'auteur des Conversations du vieillard de Vichi, qui insiste sur le principe que le désir 'd'être un jour des hommes estimables, de bons citoyens; de remplir leurs devoirs' doit être dans le cœur des enfants eux-mêmes, reprend à son compte l'image de 'la cire molle: elle prend toutes les formes que l'on sait lui donner. ${ }^{, 25}$ L'adulte mènera l'enfant par des moyens doux, mais il le mènera quand même. ${ }^{26}$

\section{Le calendrier républicain, ou l'ère de la raison}

Les calendriers sont utilisés par des gens de toutes les parties de la société, et le calendrier républicain était conçu comme un instrument pédagogique qui serait entre les mains et à la portée de tous, enfants comme adultes. Il figure dans de nombreuses œuvres pédagogiques, soit en entier, soit en forme abrégée (noms des mois et des jours). Nous avons 
$\mathrm{vu}$, dans l'iconographie et dans les écrits pédagogiques, la coexistence de différents aspects: lumière pour le monde entier, ou pour la patrie seulement; un commencement entièrement neuf, ou un retour au passé. On trouve ces différents aspects dans le calendrier républicain. Ses auteurs le voulaient logique, scientifiquement parfait, dépourvu de préjugés et de superstitions; un de ses buts était de ramener le peuple à la connaissance de la nature et à la pratique de l'agriculture. Comme les nouvelles mesures de longueur, de volume et de poids, il était en principe susceptible d'être utilisé par toutes les nations.

La perfection logique et scientifique de ce calendrier devait le distinguer du calendrier grégorien. Pour tous les deux, la division du temps la plus importante est l'année solaire. Dans notre calendrier grégorien (qui est au fond le calendrier julien, réformé en 1582 sous le pape Grégoire XIII), deux cycles se chevauchent: la semaine et le mois. Cela fait que chaque année est différente de l'année précédente, et il faut un calendrier différent chaque année. En plus, les mois sont de longueur inégale, non seulement parce que le nombre de jours dans l'année n'est pas divisible par douze, mais pour d'autres raisons aussi: comme les Romains considéraient février, consacré aux dieux de l'enfer, comme un mois néfaste, ils l'ont fait plus court que les autres mois.

Les auteurs de la Commission chargée du calendrier reprennent, en le développant, le calendrier égyptien. Ils établissent douze mois de 30 jours chacun, suivis de cinq jours complémentaires ou épagomènes à la fin, c'est-à-dire, selon le calendrier grégorien, en septembre. Ces jours, nommés Vertu, Génie, Travail, Opinion et Récompenses, sont consacrés à des fêtes patriotiques. Tous les quatre ans, il y a un sixième jour, qui fête la Révolution; et moins souvent, on fait d'autres ajustements. Chaque mois est divisé en trois décades, ou périodes de dix jours. Le principe du calendrier est donc qu'à l'exception de l'année bissextile, chaque année ressemble précisement à toute autre. On sait par exemple que le quatorze du mois tombe un quartidi. Une fois que ce système sera établi partout, une seule version du calendrier contiendra toutes les informations nécessaires pour n'importe quelle année, sauf les phases de la lune. Le temps est, à cette exception près, parfaitement cyclique.

L'année commence à l'équinoxe vrai d'automne, le 22 septembre; la nouvelle ère a commencé le 22 septembre 1792, date de la proclamation de la République. Gilbert Romme, dans son rapport, souligne cette heureuse coïncidence:

Ainsi le Soleil a éclairé à la fois les deux pôles et successivement le globe entier, le même jour où pour la première fois, a brillé dans toute sa pureté, sur la nation française, le flambeau de la liberté qui doit un jour éclairer tout le genre humain. ${ }^{27}$ 
La métaphore de la lumière est très développée dans cette phrase de Romme, avec les mots 'soleil', 'éclairer' (deux fois), 'briller', 'flambeau.' $C^{\prime}$ est la France qui apporte une lumière universelle: on parle du globe entier et de tout le genre humain. La première gravure que j'ai décrite convient parfaitement à cette phrase. Il n'y a qu'une ombre au tableau: du point de vue symbolique, c'est un peu malheureux de commencer l'année, et la République, au moment où la nuit va devenir plus longue que le jour.

Plusieurs projets de nomenclature ont été considérés, y compris une nomenclature ordinale et une nomenclature 'révolutionnaire'. Selon Pierre-Joseph Duhem, la première avait un grand avantage: 'Votre calendrier, qui n'eût été que celui de la nation française, deviendra celui de tous les peuples. Il ne s'écarteront jamais de l'ordre numérique, qui est celui de la nature. ${ }^{28}$ Dans la version qui a finalement été retenue, les noms des mois sont censés représenter ce qui se passe dans la nature à ce moment-là de l'année. Les auteurs ont voulu créer un rapport réel où non seulement la division du temps correspond aux faits astronomiques, mais aussi où le nom exprime parfaitement la chose. Fabre d'Eglantine dit dans son rapport que, dans les noms des mois, on a cherché à mettre à profit l'harmonie imitative de la langue,

de telle manière que les noms des mois qui composent l'automne [vendémiaire, brumaire, frimaire] ont un son grave et une mesure moyenne, ceux de l'hiver [nivôse, pluviôse, ventôse] un son lourd et une mesure longue, ceux du printemps [germinal, floréal, prairial] un son gai et une mesure brève et ceux de l'été [messidor, thermidor, fructidor] un son sonore et une mesure large. ${ }^{29}$

Les noms des jours, à la différence de ceux des mois, sont des numéraux, entièrement pratiques et sans contenu symbolique: primidi, duodi, tridi, quartidi, quintidi, sextidi, nonidi, décadi.

Les auteurs du calendrier républicain ont donc établi leur division du temps d'une manière qu'ils voulaient universellement valable. L'année solaire est la base du calendrier et le début de l'année est basé sur l'équinoxe vrai, pas sur une convention. La division de l'année est régulière, avec des mois de longueur égale. Le jour à son tour est divisé en dix heures de dix parties; le système décimal est donc appliqué au temps, comme aux autres mesures - $l^{\prime}$ horloge décimale devait entrer en vigueur un peu plus tard que le calendrier; elle a eu peu d'effet en pratique. ${ }^{30}$ Les noms des jours et des mois ne rappellent plus les dieux et les personnes de l'Antiquité. La semaine, qui a des racines très anciennes mais qui est surtout associée à la religion, est abolie et, en abandonnant le calendrier traditionnel, on supprime les jours des saints et les fêtes religieuses. En principe, ces deux derniers faits - la suppression de la 
semaine et des jours des saints - feraient un calendrier qui ne donne la préférence à aucune religion.

En même temps, comme nous l'avons vu, d'autres aspects font obstacle à l'adoption de ce calendrier en dehors de la France. Comme le début de l'année est défini comme minuit à l'équinoxe d'automne à Paris, le temps est centré sur la France. D'ailleurs, comme il y a des difficultés à déterminer à l'avance le moment précis de l'équinoxe quand il tombe près de minuit, on ne sait pas le jour où l'année doit commencer, de sorte que, comme le dit Lanjuinais, 'nous en sommes réduits à disputer ridiculement entre le 22 et le 23 septembre (vieux style). ${ }^{\prime 31}$ Le résultat de cela est qu'on ne sait pas à l'avance quand il y aura une année bissextile. Les noms des mois passeraient difficilement dans une autre langue ou dans un pays où le climat est différent. ${ }^{32}$ Dans le détail, le calendrier s'applique encore davantage à la France: à chaque jour, au lieu d'un saint, on associe une plante, un animal ou un outil, dans le projet de ramener la nation à l'agriculture. Évidemment ce sont des animaux, des plantes et des outils de France, placés au moment de l'année qui est approprié pour la France. Pour la première décade de germinal (fin mars), on a des plantes comme primevère, platane, asperges, tulipe; c'est trop tard pour certaines régions, trop tôt pour d'autres.

Ces difficultés liées à la partie scientifique de la division du temps sont peut-être moins importantes que son contenu politique. A part la suppression de la semaine, à laquelle on est attaché dans toute l'Europe et dans beaucoup d'autres pays, l'empêchement le plus évident à la valeur universelle du calendrier républicain est le fait de compter les ans à partir de la proclamation de la République française.

On avait voulu faire un calendrier fondé sur la raison, digne de remplacer l'ancien calendrier comme le nouveau système de mesures devait remplacer les anciens systèmes. Mais, comme nous l'avons vu, le contenu politique de ce calendrier et aussi son contenu scientifique même, qui en principe aurait dû le rendre universel, l'empêchent d'être acceptable à d'autres nations.

\section{La présentation pédagogique du calendrier}

Le calendrier républicain, comme nous l'avons dit plus haut, était conçu comme l'instrument pédagogique par excellence pour les gens de tous les âges. En plus de ses nombreuses publications à part, il paraît souvent en entier dans les livres pédagogiques. Dans d'autres cas, dans les livres pour les jeunes enfants, une version simplifiée ne présente que les mois et les jours de la décade. Mais l'intention pédagogique est une chose, la réception en est une autre: il semble que peu de gens se soient servis du 
nouveau calendrier, sauf pour les publications officielles. Les lettres au Comité d'instruction publique aux Archives nationales sont datées suivant le nouveau style, mais la correspondance avec les autorités représente peut-être un cas spécial, et en tout cas ce sont généralement les partisans les plus fervents de la Révolution qui écrivent au Comité.

La présentation du calendrier pour les adultes comprenait les deux systèmes, grégorien et républicain; les mois et les jours du calendrier grégorien se trouvent souvent à l'extrême gauche de chaque colonne. Pour les petits enfants au contraire, on ne devait enseigner $\mathrm{d}^{\prime}$ abord que le nouveau système, sans l'ancien. ${ }^{33}$ Dans plusieurs abécédaires et livres de lecture, on se sert du calendrier comme principe d'organisation. On donne un petit morceau de lecture pour chaque jour de la décade ou pour chaque décade ou mois de l'année, comme c'est le cas pour les Pensées instructives pour chaque jour de la décade, de Person, ou pour les Epîtres et Evangiles du républicain, pour toutes les Décades de l'année, de Henriquez, livre qui a connu un grand succès et qui est mentionné favorablement dans le rapport de Lakanal (33). ${ }^{34}$

La même chose est vraie pour des livres illustrés comme la Nouvelle méthode d'enseigner l'ABC de l'éditeur Devaux, qui a paru en plusieurs éditions pendant et après la Révolution. Une gravure illustre une petite histoire morale pour chaque jour de la décade. Pour nonidi, on voit '[l]a vieillesse', où le texte dit que '[l]'âge avancé mérite des égards et du respect', et pour décadi, c'est '[1]es oiseaux rendus à la liberté', l'histoire d'un garçon qui prenaient des oiseaux et puis les lâchait, disant: '[s]oyez libres. $^{, 3}$

Le Premier livre républicain de Macarel, qui reçoit une mention dans le jugement du jury, donne lui aussi une petite gravure accompagnée d'un court passage pour chaque jour de la décade. ${ }^{36}$ Une autre gravure pour chaque saison, avec les noms des mois selon le nouveau calendrier, est accompagnée d'un passage plus long. Pour l'été, on voit un garçon assis sous un arbre avec un jeune parent, qui est instituteur. Le texte est une autre version de l'anecdote des oiseaux et, dans la gravure, le garçon vient de les laisser s'envoler. Au fond du tableau, on voit des travaux de la saison, et au plan moyen un jeune enfant nage dans la rivière, 'comme on se noie, lorsqu'on ne sait pas nager' - un projet fixé par le Comité était de faire apprendre la natation aux enfants dans les écoles. En principe, donc, les enfants devaient apprendre les rudiments du nouveau calendrier en même temps qu'ils apprenaient les phénomènes naturels, les saisons, les travaux saisonniers, et les vertus républicaines. C'est ainsi que la nouvelle mesure du temps devait entrer dans la structure même du monde pour la jeune génération. 


\section{Conclusion}

Certains auteurs d'œuvres soumises au concours de livres scolaires se tiennent à l'écart des événements politiques, que ce soit par inclination, par prudence, ou par la nature de la matière traitée. Mais d'autres sont fortement marqués par le moment, et il y en a qui n'auraient jamais pris la plume si ce n'était pour les circonstances extrêmes. C'est chez les partisans fervents de la Révolution que le lecteur est ébloui, sinon toujours par l'éclat de leur logique et de leur style, du moins par la fréquence des allusions à la lumière et au jour nouveau: feu, aurore, soleil, flambeau, briller, éclairer. Déjà une longue tradition représentait la lumière triomphant de l'obscurité. Les écrits et images dont il s'agit ici, tout en suivant cette tradition, précisent le moment de la venue de la lumière. A les en croire, le soleil, annoncé depuis quelque temps, ne s'est vraiment levé qu'avec la Révolution.

Mais, comme nous l'avons vu, la relation entre le passé, le présent et l'avenir est plus complexe que ces expressions ne le feraient croire. On cherche des modèles dans l'Antiquité pour construire la cité nouvelle, et l'autorité des parents et des enseignants est soutenue par la plupart des auteurs, même si certains prônent plus de liberté pour les enfants. Rigaud et l'auteur du Vieillard de Vichi, par exemple, veulent que parents et maîtres usent de douceur et de finesse psychologique pour mener les enfants sur la bonne voie. Mais il faut les mener; les enfants, porteurs d'avenir, ne peuvent pas être élevés dans le vide. La plupart des auteurs évitent de parler des conflits possibles entre la nature et la raison, et ceux qui abordent la question la traitent de façon sommaire. La raison suggère-t-elle qu'un enfant doit peu d'égards à un père méchant? L'auteur de la Véritable civilité républicaine tranche, d'une manière qui suit plutôt la tradition: 'qu'importe!' (68). Il y a également une tension en ce qui concerne l'étendue du nouveau jour. Brille-t-il sur la France seulement, ou sur la terre entière? Les deux idées sont fréquentes.

Quelques-unes des mêmes questions et des mêmes tensions se trouvent dans le calendrier républicain. Pour établir un calendrier basé sur la nature, on est obligé de faire un choix entre les divisions de temps qui se trouvent dans la nature même. Milo observe que la Commission chargée du calendrier, ayant choisi l'année solaire, aurait dû selon sa propre logique commencer par diviser l'année en quatre saisons marquées par les équinoxes et les solstices; or c'est la division traditionnelle en douze mois qui a été choisie, parce que les gens y étaient habitués (204). La Commission retournait à un modèle tiré de l'Antiquité, qui, comme le calendrier grégorien, comprend douze mois. Il n'est pas si facile de se débarrasser des habitudes enracinées. Quant à la décade, selon Milo, elle est aussi 'fausse' que la semaine qu'elle était faite pour 
remplacer. Ni n'une ni l'autre ne correspondent à rien dans la nature, et l'adoption de la décade témoigne de 'la primauté du rationnel sur le naturel' (210).

On visait un découpage du temps qui, étant basé sur la nature et la raison, serait valable pour toute la terre. Mais la nomenclature 'naturelle' des mois est limitée à la France. Le début de l'année et celui de l'ère sont basés sur la position géographique de la France et sur des événements politiques qui séparent les gens au lieu de les unir. Dans l'exécution du projet, on a construit un système qui était centré sur la France dans son contenu politique et dans son essence scientifique même. Comme Milo l'a indiqué, le calendrier républicain, produit d'une Commission travaillant dans un 'possibilisme à grande échelle' (194), semble d'abord accorder la nature et la raison en écartant toute division fausse du temps. Mais enfin c'est un produit qui suit dans ses différents aspects la nature, la raison ou la tradition. Nous pourrions peut-être en dire autant des différentes propositions pour l'éducation des enfants: là aussi, les auteurs essaient de créer un nouveau temps humain, et là aussi, ils suivent à différents moments la nature, la raison et la tradition.

\section{NANCY SENIOR}

Université de la Saskatchewan

\section{Notes}

1 Pour une bibliographie des écrits pédagogiques et des livres pour enfants, voir H.C. Harten, Les écrits pédagogiques sous la Révolution (Paris: Institut National de Recherche Pédagogique, 1989); et M. Manson, Les livres pour l'enfance et la jeunesse sous la Révolution (Paris: Institut National de Recherche Pédagogique, 1989). Pour les œuvres du concours, voir J. Guillaume, 'Livres élémentaires de la première république', Le dictionnaire de pédagogie, vol. II (1882); J.-F. Chassaing, 'Les manuels d'enseignement primaire de la Révolution et les idées révolutionnaires', J. Morange et J.-F. Chassaing, Le mouvement de réforme de l'enseignement en France 1760-1798 (Paris: P.U.F., 1974); J. Hébrard, 'La Révolution expliquée aux enfants: les catéchismes de l'An II', M.-F. Lévy (sous la dir. de), L'enfant, la famille et la Révolution française (Paris: Olivier Orban, 1990) 171-92; N. Senior, "Toute une génération élevée par lui": Rousseau et les livres scolaires sous la Révolution', Etudes Jean-Jacques Rousseau 9 (1997) 21-33; et N. Senior, 'The Teaching of Reading during the Revolution', Studies on Voltaire and the eighteenth century 302 (1992) 379-407.

Pour le calendrier, voir Service des calculs et de mécanique céleste du Bureau des longitudes, Le calendrier républicain, de sa création à sa disparition, suivi d'une concordance avec le calendrier grégorien (Paris: Bureau des Longitudes, 1994); Paul Couderc, Le calendrier, 5e édition (Paris: P.U.F., 1981); Francesco Maiello, Histoire du calendrier. De la liturgie à l'agenda (Paris: Seuil, 1993); Daniel S. Milo, Trahir le 
temps (histoire) (Paris: Les Belles Lettres, 1991); et Claire Gaspard, 'Les almanachs de l'an II: quoi de neuf en dehors du calendrier?', Annales historiques de la Révolution françaises 264 (1986) 141-59.

2 Condorcet, Moyens d'apprendre à compter sûrement et avec facilité, 2e édition (Paris, an VIII); De Wailly, Moyens de faciliter la lecture, et de rendre uniformes la prononciation et l'orthographe, AN F17 11648; Lhomond, Elements de la grammaire françoise (Paris, 1790); Volney, La loi naturelle, édition critique par Gaston-Martin, 1793 (Paris, 1934). Le livre (posthume) de Condorcet et celui de Lhomond ont été couronnés. Ceux de De Wailly et de Volney n'étaient pas assez simples pour être des livres élémentaires. Voir le Jugement du Jury des livres élémentaires présentés au Concours ouvert par la loi du neuf pluviôse, an deux, AN F17 1331 B; et Lakanal, Rapport fait au Conseil des Cinq-Cents [...] sur les livres élémentaires [...] (Paris, an IV).

3 Un rapport pour le Comité observe que beaucoup d'ouvrages sur la morale présentés au concours sont 'marqués au coin de la médiocrité' (Rapport, 1ère section, Bureau des Livres Elémentaires, AN F17 1331 B). L'auteur observe que 'tous les esprits ont senti le besoin de recréer les mœurs en même temps que les lois', mais que l'exécution demande un 'homme supérieur' pour remonter aux principes et en tirer les conséquences (Lakanal, Rapport 31). Le style des œuvres dans les différentes catégories n'est pas toujours raffiné et l'orthographe laisse à désirer non seulement dans les manuscrits, mais quelquefois même dans les imprimés. Plusieurs ont même été écrites avant la Révolution et soumises au concours sous forme d'imprimés datant de quelques années. D'autres, composées exprès pour le concours, se concentrent sur la matière en question mathématiques, grammaire, sciences - et laissent de côté les questions politiques autant que possible. Je ne traite ici que de celles qui comprennent le nouveau calendrier ou qui mentionnent directement ou indirectement les événements récents.

4 Hébrard signale le grand nombre d'auteurs de trois professions: les prêtres anciens régents, les administrateurs locaux ou nationaux, et les imprimeurs (180). Parmi les imprimeurs, on peut mentionner Devaux. L'auteur dont les livres ont provoqué la remarque sur la médiocrité des œuvres soumises au concours est administrateur. Ces trois catégories s'appliquent surtout aux auteurs d'imprimés; pour les manuscrits, ou pourrait ajouter les instituteurs et institutrices, et les militaires, dont quelques-uns indiquent aussi leur profession dans la vie civile.

5 Pierre-Platon Blanchard, Catéchisme de la nature, ou Religion et morale naturelles, nouvelle édition (Paris, an III).

6 Renaud, Maximes générales, AN F17 11648. Nyon le jeune, dans son Catéchisme républicain, ou Principes de la constitution française (Paris, an II), dit que le gouvernement démocratique est 'le seul qui soit juste, naturel et vraiment digne $d^{\prime}$ un peuple libre. Telle est la République, existant en France actuellement' (7). Selon Hébrard, ce livre d'un éditeur parisien ne paraît pas avoir été soumis au concours, mais il est inscrit 'dans le didactisme qui est à l'origine du concours de l'an II' (voir 182-83).

7 La citoyenne Lambert, Avis aux jeunes republicaines françoises, AN F17 1331 B, 4e maxime.

8 Prévost, Education indispensable aux hommes libres et à leurs enfans (Paris, an III) 8.

9 Levesque, Cours élémentaire de morale, ou Le père instituteur de ses enfants (Paris, an V) 36 . 
10 Instruction élémentaire sur la morale républicaine, s.d., AN F17 11648, 3. (Comme c'est le titre d'une catégorie du concours, plusieurs livres se servent de ce même titre, avec le mot instruction soit au singulier, soit au pluriel.)

11 La citoyenne Desmarest, Premiers éléments de l'instruction républicaine (Paris, an II) 6.

12 Cavaillon, Discours prononcé à la société des sans-culottes d'Excideuil, AN F17 11648, non paginé.

13 Desparens, Principes élémentaires d'éducation républicaine (Paris, s.d) 5.

14 Collignon-Dumont, l'instituteur du peuple, ou la vérité sans voile 2 (s.d).

15 Cavaillon, série de dialogues, sans titre, AN F17 11648.

16 Taboureau, Catéchisme politique, moral, et philosophique a l'usage des vrais républicains, non paginé (an 3) AN F17 11648, n.p.

17 Instructions élémentaires sur la morale républicaine, an II, AN F17 11648, 1.

18 Evangile des républicains, précédé du rapport fait par le citoyen Fabre d'Eglantine, sur le nouveau Calendrier décrété par la Convention Nationale (Paris, an II) 74.

19 Fréville, le temple de la morale, ou pensées gnomiques (Paris, an III); et Fréville, Vie et mort républicaines du petit Emilien (Paris, an II).

20 Godineau, Syllabaire républicain, s.d., AN F17 11648, 54-55.

21 Prévost, Véritable civilité républicaine à l'usage des jeunes citoyens des deux sexes (Paris, an III) 67-68.

22 Guérin-Albert, Avis aux jeunes républicaines françaises, s.d., 6-7.

23 Aubert, Etudes sur l'éducation (Paris, 1792) 20.

24 Rigaud, Dialogues sur la morale et les moyens de rendre les hommes heureux et Suite des dialogues sur la morale, AN F17 11648, 29.

25 Conversations du vieillard de Vichi (Paris, an II) 4-5.

26 Pour une discussion plus complète des rapports entre les enfants et leurs parents et instituteurs dans les œuvres du concours, voyez mon article 'Toute une génération élevée par lui' (note 1).

27 Rapport cité dans Le calendrier républicain 23.

28 Cité par Milo 215.

29 Rapport fait à la Convention Nationale, dans la séance du 3 du second mois de la seconde année de la République Française [...], L'evangile des républicains, 23.

30 Le jour, qui pourrait aussi bien être divisé en dix parties qu'en douze ou vingt-quatre, était le moindre des soucis des réformateurs, car les heures étaient moins fortement empreintes de signification religieuse que les jours. On a fait moins d'effort pour faire accepter l'horloge décimale que pour le calendrier républicain.

31 Opinion de Lanjuinais, Le calendrier républicain 92; voir aussi 13-14.

32 Les noms de mois sont inspirés non seulement par leur son, mais aussi et surtout pour leur contenu sémantique en français, à partir du latin: floréal, mois des fleurs; pluviôse, mois de la pluie. Ils décrivent les saisons en France; selon Lanjuinais, 'Les nouveaux noms des mois sont vérité dans le nord, et perpétuel 


\section{Nancy Senior}

mensonge dans le midi' (Opinion de Lanjuinais sur l'introduction du calendrier des tyrans dans la Constitution républicaine, an III, Le calendrier républicain 92).

33 Selon Nyon le jeune dans son Catéchisme républicain, 'dans sa régénération complette, il falloit abolir jusqu'au souvenir de l'esclavage et de la tyrannie'. L'ancien calendrier, 'inventé dans des tems de servitude et de despotisme, réformé par le fanatisme et la superstition', doit être entièrement remplacé par le nouveau calendrier.

34 Person, Pensées instructives pour chaque jour de la décade, s.l., 1794; Henriquez, Epîtres et évangiles du républicain, pour toutes les Décades de l'année, à l'usage des jeunes sans-culottes, nouvelle édition (Troyes, s.d).

35 Nouvelle méthode d'enseigner l'ABC, éd. Devaux (Paris, an II).

36 Macarel, Premier livre républicain (Paris, an II). 